\title{
ON THE EVIDENCE FOR EXTREME GRAVITY EFFECTS IN MCG -6-30-15
}

\author{
K. A. Weaver ${ }^{1}$ and T. Yaqoob ${ }^{2}$
}

\begin{abstract}
We examine the unusual Fe-K $\alpha$ line profile in MCG -6-30-15 observed by $A S C A$ during a deep minimum in the source intensity. The intense red wing and depressed blue wing of the line have been interpreted as evidence for extreme gravitational redshifts in terms of emission from within six gravitational radii of a black hole. We find that the data do not uniquely support this interpretation and can be equally well explained by occultation of the continuum source and the putative line-emitting accretion disk, which we offer as an alternative hypothesis. Two problems with previous modeling were that the equivalent width of the line during the deep minimum was required to be unusually large $(>1 \mathrm{keV})$ and the line intensity was thought to increase as the source became dim. The occultation model does not suffer from these problems. Our results serve to highlight the hazards of over-interpreting observational results which have low statistical significance, to the extent that theoretical implications can become generally accepted when the data do not provide a strong case for them.
\end{abstract}

Subject headings: black hole physics - galaxies:active galaxies:individual:MCG-6-30-15 - line:profiles - X-rays:galaxies

\footnotetext{
${ }^{1}$ Department of Physics and Astronomy, Johns Hopkins University, Bloomberg Center, Baltimore, MD 21218. kweaver@pha.jhu.edu

${ }^{2}$ NASA/ Goddard Space Flight Center, Laboratory for High Energy Astrophysics, Greenbelt, MD 20771, USA.
} 


\section{Introduction}

Recently, Iwasawa et al. (1996; hereafter I96) found an extremely broad iron $\mathrm{K} \alpha$ emission line with a particularly prominent red wing, extending down to $\sim 4 \mathrm{keV}$, during an extended observation with the Advanced Satellite for Cosmology and Astrophysics (ASCA; see Tanaka, Inoue \& Holt 1994). The peculiar line profile, measured during what has been dubbed a 'deep minimum' (or DM) in the X-ray light curve, had much more emission in the red wing, and much less around the line rest energy $(\sim 6.4 \mathrm{keV})$, compared to the Fe K $\alpha$ profiles thus far measured for MCG -6-30-15 and other AGNs (e.g. Tanaka et al. 1995, Yaqoob et al. 1995, Nandra et al. 1997). Also, the equivalent width of the line was unusually large, of the order of $\sim 1 \mathrm{keV}$, a factor of $\sim 3$ larger than most such measurements of broad iron K lines in Seyfert 1 galaxies. For the DM state of MCG -6-30-15, I96 rejected the standard model for the Fe-K $\alpha$ line profile (e.g. see Fabian et al. 1989), in which the line is emitted in an accretion disk rotating about a Schwarzschild black hole, with the inner disk extending no closer than the radius for marginally stable orbits, (i.e. 6 gravitational radii, or $6 r_{g}$ ). Instead, the extreme gravitational redshifts implied by the bloated red wing of the line were explained either in terms of emission from inside the last stable orbit around a Schwarzschild hole (Reynolds \& Begelman 1997) or emission in a disk rotating about a Kerr black hole (e.g. I96; Dabrowski et al. 1997; Bromley, Miller \& Pariev 1998). In the latter case the last stable orbit extends down to $1.24 r_{g}$ for a maximally rotating Kerr black hole. In both cases, the red wing of the line is accounted for by emission closer to the black hole event horizon, so the photons can experience the effects of very strong gravity, resulting in extremely large redshifts.

We point out that in terms of fitting the DM data for MCG -6-30-15 with the standard model (Schwarzschild hole, disk extending to $6 r_{g}$ ) and the Kerr model (disk extending to $1.24 r_{g}$ ), the largest difference in the fitting statistic, $\Delta \chi^{2}$, is 6.2 for the same number of free model parameters (see rows 1 and 3 in Table 3 of I96 who assume a disk inclination of $30^{\circ}$ and an outer radius of $15.5 r_{g}$ ). While this may be interpreted as being formally statistically significant, $A S C A$ spectral fits do not in general (and in this case, in particular) include the effects of systematic errors which could reduce the overall significance of the result. Since the implications of really being able to observe X-rays inside

of $6 r_{g}$ and even closer to a black hole event horizon are so far reaching (e.g. see Fabian 1997) it is important to investigate the robustness of the result for MCG -6-30-15, the only case thus far reported. 


\section{ASCA Data}

ASCA observed MCG -6-30-15 for over 4 days starting 1994, July 23. ASCA has four identical, thin-foil, light-weight X-ray telescopes (XRT) which focus X-rays onto one of two Solid-state Imaging Spectrometers (SIS) or one of two Gas Imaging Spectrometers (GIS, see Ohashi et al. 1991). See Tanaka et al. (1994) for a summary of the ASCA mission and focal-plane detectors. The SIS sensors, each one consisting of four CCD (charge coupled device) chips were operated in a mode in which only one chip was exposed (1-CCD mode) and the data were accumulated in FAINT mode. Hereafter the two SIS sensors are referred to as SIS0 and SIS1 and the two GIS sensors as GIS2 and GIS3. The data reduction and selection criteria are similar to those described in Yaqoob et al. (1994).

The lightcurve of the entire observation has been presented elsewhere (I96; Reynolds et al. 1997; Yaqoob et al. 1997). We use exactly the same time intervals defined by 196 to extract spectra of the DM state (interval $i-7$ in their Figure 2) and the flare state (interval $i-3$ ), as well as the average (total) spectrum. For the DM, we obtained $3-10 \mathrm{keV}$ count rates in the range 0.13 to 0.16 counts $\mathrm{s}^{-1}$ and exposure times in the range 13.2 to $13.3 \mathrm{ks}$ for the four instruments. Figure 1 shows the ratio of the data in the DM to the best-fitting power-law model $\left(\Gamma=1.92, N_{\mathrm{H}}=1.1 \times 10^{22} \mathrm{~cm}^{-2}\right)$ using data only in the energy ranges $2-3 \mathrm{keV}$ and $8-10 \mathrm{keV}$. The excess above the underlying power-law is due to the Fe-K $\alpha$ line emission. As pointed out by I96, the emission on the blue side of the line is unusually diminished compared to the red side. The portion of the lightcurve containing the DM state is shown in Figure 2.

\section{Standard Disk-line Spectral Fits}

Using data between 3 and $10 \mathrm{keV}$ from all four instruments, we fitted the Fe-K $\alpha$ line for the average, flare and DM spectra with our 'baseline' model in which the line photons are emitted in a disk rotating around a central Schwarzschild black hole (e.g., Fabian et al. 1989). The parameters are $\theta_{\text {obs }}$ (inclination angle of the disk normal relative to the observer), $r_{i}$ (inner disk radius), $r_{o}$ (outer disk radius), $q$ (power-law index characterizing the line emissivity as $\left.\propto r^{-q}\right), I_{\mathrm{D}}$ (line intensity), and $E_{D}$ (line energy in the disk rest frame).

The inner radius, $r_{i}$, was fixed at $6 r_{g}$ where $r_{g} \equiv G M / c^{2}$ (i.e. the last stable orbit). There is interplay between $E_{D}$ and the other line parameters, so $E_{D}$ was fixed at $6.4 \mathrm{keV}$ in the rest frame, corresponding to fluorescence in a cold disk. The results are shown in Table 1, models SH1, SH2, and SH3.

We repeated the above fitting, but this time replacing the Schwarzschild black hole 
with a maximally rotating Kerr black hole (see e.g., Laor 1991). Now the inner radius is fixed at $1.24 r_{g}$, the minimum value, or last stable orbit for the Kerr metric. The spectral fit was repeated with $E_{D}$ fixed at $6.7 \mathrm{keV}$ (corresponding to He-like Fe). For the average and flare spectra, a Kerr profile does not improve the fit, but for the DM, the Kerr model provides a statistically better fit than the Schwarzschild model $\left(\Delta \chi^{2} \sim 5\right)$. The results for the DM are shown in Table 1, models KR1 and KR2. The basic reason why the Fe-K $\alpha$ line during the DM is better described by emission from matter closer than $6 r_{g}$ is that the observed strength of the blue peak of the line is too small for the observed strength of the red wing. This is compensated by the extra gravitational redshifts obtained by placing the emitter closer to the black hole, increasing the intensity of the red wing relative to the blue. Our results confirm those of I96, although the best-fitting parameters differ slightly because we fix $q$ at 2.5 and allow $\theta_{\text {obs }}$ and $r_{o}$ to be free, the contrary to their technique.

\section{Spectral Fits with an Occultation Model}

We tested whether we could fit the DM Fe-K $\alpha$ line profile without placing lineemitting matter closer to the black hole than $6 r_{g}$ by obscuring part of the standard Schwarzschild disk with optically-thick matter. Figure 3 illustrates how different parts of a Schwarzschild disk affect the Doppler and gravitational shift of line photons. Defining $g=E$ (observed) $/ E$ (emitted) (including both Doppler and gravitational effects), the shaded part labeled blue corresponds to $g>1$ and the shaded part labeled red corresponds to $g<(1 / 1.2)$. In our simple-minded model, the occultation of the disk is in the form of a strip running along the $x$ or $y$ directions, with half-width $y_{0}$ or $x_{0}$ respectively, passing symmetrically over the center of the disk. Thus, line emission from either $|y|<y_{0}$ or $|x|<x_{0}$ is blocked from view and the predicted line profiles for the two cases are shown in Figures $4 \mathrm{a}$ and $4 \mathrm{~b}$ respectively (for several values of the half-width). We use this model as a hypothesis, since we do not know the geometry of the optically-thick clouds (there are plenty of arguments for their existence, e.g. Guilbert \& Rees 1988). The clouds could be spherical, filament-shaped, or neither. Our simple model in some sense mimics an optically-thick cloud, whatever its shape, moving over the disk, resulting in some kind of average line profile. Clearly, the profiles in Figure 4a, in which much of the blueshifted emission is obscured, are relevant for the DM data.

For the spectral fits with the obscuration model, we fix $E_{D}=6.4 \mathrm{keV}$ and $r_{i}=6 r_{g}$ as before, and $r_{0}=18.5 r_{g}$ from the Schwarzschild fit (Table 1, SH3). The best-fitting parameters are shown in Table 1, model OC1. This fit is marginally better than the Kerr model with $E=6.4 \mathrm{keV}$ (model KR1) and marginally worse than the Kerr model with 
$E=6.7 \mathrm{keV}$ (model KR2) and so we conclude that the occulted, Schwarzschild disk-line model describes the spectrum during the DM just as well as the standard Kerr model.

The above fits include only Galactic absorption because we assume that there is no significant absorption from the 'warm absorber' present above $3 \mathrm{keV}$ that will affect the shape of the continuum (Fabian et al. 1994; Reynolds et al. 1995; Otani et al. 1996). This was also the assumption made by 196 and is based on observations of the warm absorber in MCG-6-30-15 in higher flux states. However, if absorption were present, this could also affect measurements of the Fe-K $\alpha$ line. Figure 5 shows the data/model ratio when the DM spectrum is fitted with Galactic absorption and a power law with the best-fitting photon index of the average spectrum $(\Gamma=1.98)$. The deficiency of low-energy photons due to the ionized absorber is significant, even above $3 \mathrm{keV}$, and implies that the flattening of the continuum in the DM (Table 1, models SH3, KR1, KR2, OC1) is due to absorption. Thus, the intrinsic spectral slope during the DM is not required to change.

We therefore fitted the DM spectrum again with the occultation-line model with $\Gamma$ fixed at the average value of $\Gamma=1.98$, but with free absorption (Table 1 , model OC2). The

number of free parameters is the same as before, but $\Delta \chi^{2}=5.7$ compared to the best-fitting Kerr model (model KR2). The occultation description of the DM is thus as viable as the scenarios posited by I96, Reynolds \& Begelman (1997), Dabrowski et al. (1997), and Bromley et al. (1998).

\section{Discussion and Conclusions}

We have examined the Fe-K $\alpha$ line profile during the deep minimum (DM) flux state for MCG-6-30-15 with an 'occultation model' in which the continuum source and much of the blueshifted part of the emission from the disk is obscured by an optically-thick cloud. This model describes the observed line profile as well as the Kerr black-hole/disk model, but does not require emission from inside $6 r_{g}$ (the last stable orbit for a Schwarzschild black hole). One difficulty with the Kerr interpretation proposed by 196 and others is that it doesn't explain why Kerr effects should only manifest themselves in the DM. The occultation model does not suffer from this problem. Also, the line intensity is not required to increase relative to the average line intensity, as has been previously claimed. Nor is the equivalent width (EW) of the Fe-K line during the DM required to be unusually large. We obtain $\mathrm{EW} \sim 400 \mathrm{eV}$ for the $\mathrm{DM}$ and flare-states, and $\mathrm{EW} \sim 470 \mathrm{eV}$ for the average line.

In addition to the optically-thick obscurer that blocks most of the continuum region, the data above $3 \mathrm{keV}$ are affected by the O VIII edge of the warm absorber that becomes 
stronger in the low-flux state (Otani et al. 1996). We measure an additional column density of $\sim 2 \times 10^{22} \mathrm{~cm}^{-2}$ in the DM, which is a factor of $\sim 2$ increase compared to the high flux state (Fabian et al. 1994). The increase in column density is consistent with a picture in which the material that is photoionized by the central source becomes less opaque to soft $\mathrm{X}$-rays due to a reduction in ionization when the continuum is blocked. Note that the extra absorption we directly measure is not necessarily related to the optically-thick clouds responsible for obscuring the $\mathrm{Fe}-\mathrm{K} \alpha$ line emission.

To conclude, we have shown that the unusual Fe-K $\alpha$ line profile in MCG $-6-30-15$ during a deep minimum in intensity does not necessarily require line emission from within $6 r_{g}$ and that the data are not adequate enough to distinguish between the Schwarzschild and Kerr solutions. Sulentic et al. (1998) have also made the suggestion that a Kerr black hole is not required, proposing that the true shape of the $\mathrm{Fe}-\mathrm{K} \alpha$ line is a double Gaussian, but offer no physical model. We have shown that the deep-minimum line profile can be explained by occultation of a standard Schwarzschild disk. This model is consistent with the detailed temporal profile of the continuum intensity dip. Our results serve to highlight the hazards of over-interpreting observational results which have low statistical significance, to the extent that theoretical implications can become generally accepted when the data do not provide a strong case for them. 


\section{REFERENCES}

Bromley, B. C., Miller, W. A., \& Pariev, V. I. 1998, Nature, 391, 54

Dabrowski, Y., Fabian, A. C., Iwasawa, K., Lasenby, A. N., \& Reynolds, C. S. 1997, MNRAS, 288, L11

Fabian, A. C., Rees, M. J., Stella, L., \& White, N. E. 1989, MNRAS, 238, 729

Fabian, A. C., et al. 1994, PASJ, 46, L59

Fabian, A. C. 1997, A\&G, 38, 10

Guilbert, P. W., \& Rees, M. J. 1988, MNRAS, 233, 475

Iwasawa, K., et al. 1996, MNRAS, 282, 1038

Laor, A. 1991, ApJ, 376, 90

McKernan, B., \& Yaqoob, T. 1998, ApJ, in press astro-ph/9804265

Nandra, K., George, I. M., Mushotzky, R. F., Turner, T. J. , \& Yaqoob, T. 1997, ApJ, 476, 70

Ohashi, T. et al. 1991, Proc. SPIE, 1549, 9

Otani, C. et al. 1996, PASJ, 48, 211

Reynolds, C S., Fabian, A. C., Nandra, K., Inoue, H., Kunieda, H., \& Iwasawa, K. 1995, MNRAS, 277, 901

Reynolds, C. S., \& Begelman, M. C. 1997, ApJ, 488, 109

Sulentic, J. W., Marziani, P., Zwitter, T., Calvani, M., Dultzin-Hacyan, \& D. 1998, ApJ, in press, astro-ph/9712336

Tanaka, Y., Inoue, H., \& Holt, S. S. 1994, PASJ, 46, L37

Tanaka, Y., et al. 1995, Nat, 375, 659

Yaqoob, T., Serlemitsos, P. J., Mushotzky, R. F., Madejski, G., Turner, T.J., \& Kunieda, H. 1994, PASJ, 46, L173

Yaqoob, T., Edelson, R., Weaver, K. A., Warwick, R. S., Mushotzky, R. F., Serlemitsos, P. J., \& Holt, S. S. 1995, ApJ, 453, L81

Yaqoob, T., McKernan, B., Ptak, A., Nandra, K., Serlemitsos, P. J. 1997, ApJ, 490, L25 
Table 1. MCG-6-30-15: Relativistic Disk-Model Spectral Fits $(3-10 \mathrm{keV})$ for the Fe K $\alpha$ line ${ }^{1}$.

\begin{tabular}{|c|c|c|c|c|c|c|c|c|}
\hline Data set & Model $^{2}$ & $\Gamma$ & $r_{o} / r_{g}$ & $\begin{array}{c}\theta_{\text {obs }} \\
\text { (degrees) }\end{array}$ & $I_{\mathrm{Fe}}{ }^{3}$ & $\begin{array}{l}\mathrm{EW} \\
(\mathrm{eV})\end{array}$ & $y_{0} / r_{g}$ & $\chi^{2} / \nu$ \\
\hline Average & SH1 & $1.98_{-0.04}^{+0.04}$ & $15.6_{-4.4}^{+5.7}$ & $33.7_{-1.9}^{+1.8}$ & $1.88_{-0.31}^{+0.30}$ & $473_{-78}^{+75}$ & $\cdots$ & $1417.9 / 1355$ \\
\hline Flare & SH2 & $2.00_{-0.06}^{+0.07}$ & $6.1_{-0.01 \dagger}^{+2.0}$ & $39.4_{-6.5}^{+2.4}$ & $2.29_{-0.80}^{+0.78}$ & $410_{-140}^{+142}$ & $\ldots$ & $925.6 / 899$ \\
\hline Deep Min. & SH3 & $1.71_{-0.17}^{+0.17}$ & $18.5_{-9.3}^{+19.4}$ & $27_{-10}^{+10}$ & $1.52_{-0.68}^{+0.74}$ & $665_{-298}^{+324}$ & $\ldots$ & $305.2 / 323$ \\
\hline Deep Min. & KR1 & $1.73_{-0.17}^{+0.19}$ & $19.8_{-18.56 \dagger}^{+30.0}$ & $31_{-12}^{+39}$ & $2.28_{-0.95}^{+1.04}$ & $1070_{-446}^{+488}$ & $\ldots$ & $299.6 / 323$ \\
\hline Deep Min. & $\mathrm{KR} 2^{4}$ & $1.73_{-0.18}^{+0.21}$ & $13.3_{-12.06+}^{+15.4}$ & $27_{-10}^{+39}$ & $2.42_{-1.04}^{+1.22}$ & $1130_{-486}^{+570}$ & $\ldots$ & $297.6 / 323$ \\
\hline Deep Min. & $\mathrm{OC} 1^{5}$ & $1.74_{-0.17}^{+0.20}$ & $18.5 \mathrm{f}$ & $46_{-23}^{+43}$ & $1.63_{-0.71}^{+1.07}$ & $481_{-210}^{+316}$ & $8.0_{-8.0}^{+4.1}$ & $298.2 / 323$ \\
\hline Deep Min. & $\mathrm{OC} 2^{5,6}$ & $1.98 \mathrm{f}$ & $18.5 \mathrm{f}$ & $51_{-24}^{+38}$ & $1.53_{-0.76}^{+0.78}$ & $404_{-201}^{+206}$ & $8.8_{-7.2}^{+4.1}$ & $291.9 / 323$ \\
\hline
\end{tabular}

${ }^{1}$ Errors are $90 \%$ confidence for 4 interesting parameters $\left(\chi^{2}+7.78\right)$. Fixed parameters are denoted by ' $\mathrm{f}$ '. The center energy of the iron line in the disk frame is fixed at $6.4 \mathrm{keV}$ unless otherwise stated. $N_{\mathrm{H}}$ (Gal.) $=5 \times 10^{20} \mathrm{~cm}^{-2}$ is assumed for all fits.

${ }^{2}$ Theoretical models for the iron line are SH: Schwarzschild profile, KR: Kerr profile, OC: occulted, Schwarzschild disk-line model.

${ }^{3}$ Line intensity in units $10^{-4}$ photons $\mathrm{cm}^{-2} \mathrm{~s}^{-1}$. Intensities and equivalent widths $(\mathrm{EW})$ are in the observed frame.

${ }^{4}$ The iron line center energy in this Kerr model is fixed at $6.7 \mathrm{keV}$ in the disk frame.

${ }^{5}$ The occulted disk model is described in the text. The parameter $y_{0}$ is the half-width of an obscuring strip running symmetrically over the center of the disk (see Figure 3 ).

${ }^{6}$ In this model there is an additional absorbing column with $N_{H}=1.96_{-1.31}^{+1.29} \times 10^{22} \mathrm{~cm}^{-2}$.

${ }^{\dagger}$ The lower bounds on $r_{o}$ for these fits are not true statistical errors but represent the radius of last stable orbit around a maximally rotating Kerr black hole $\left(1.235 r_{g}\right)$. 


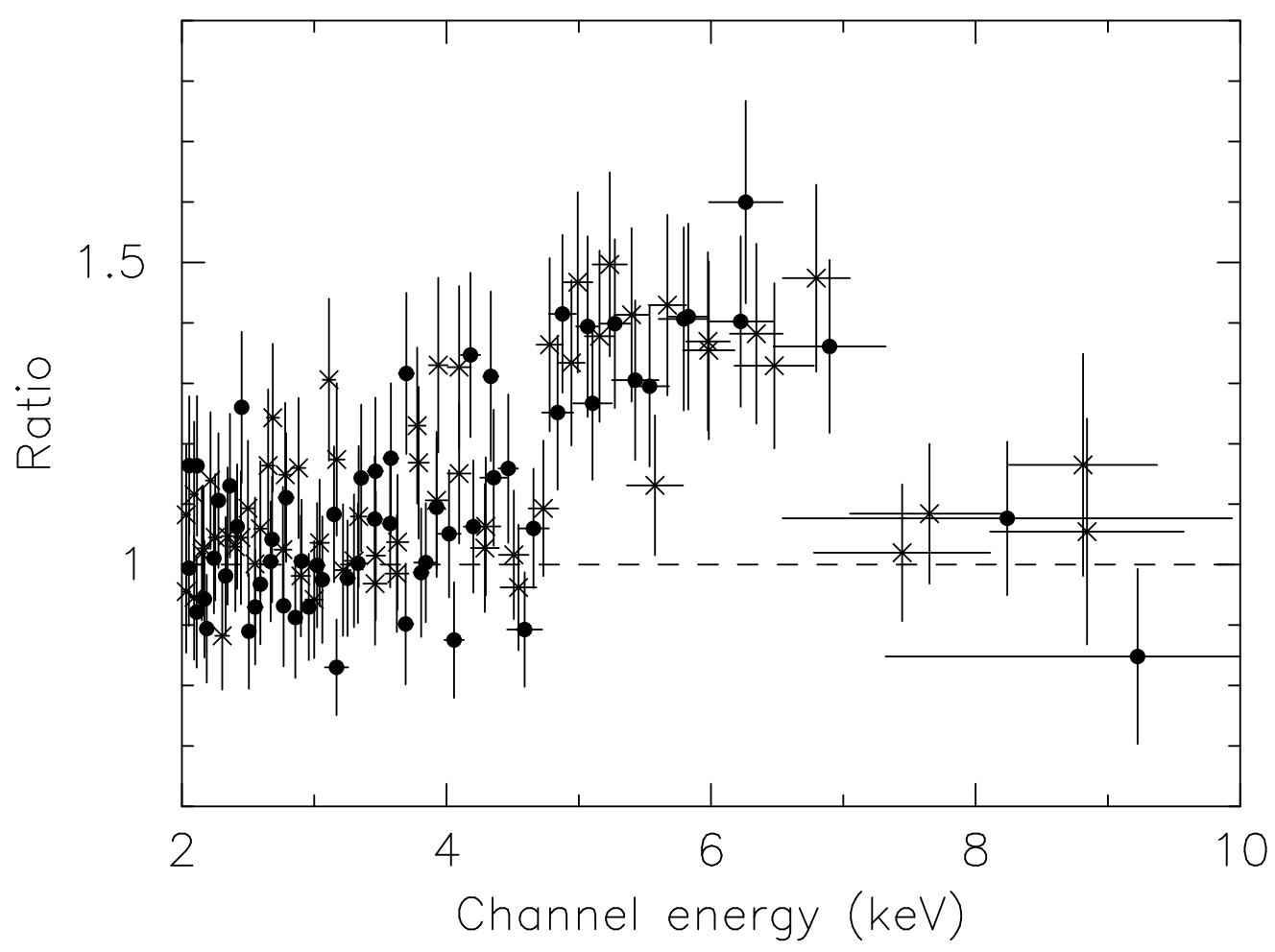

Figure 1

Fig. 1. - Ratio of the $A S C A$ data (all detectors) from the deep minimum to a continuum model that consists of the best-fitting absorbed power law. The data between 3 and 8 $\mathrm{keV}$ were ignored for the fit. Filled circles correspond to SIS0 and SIS1 data and crosses correspond to GIS2 and GIS3 data. 


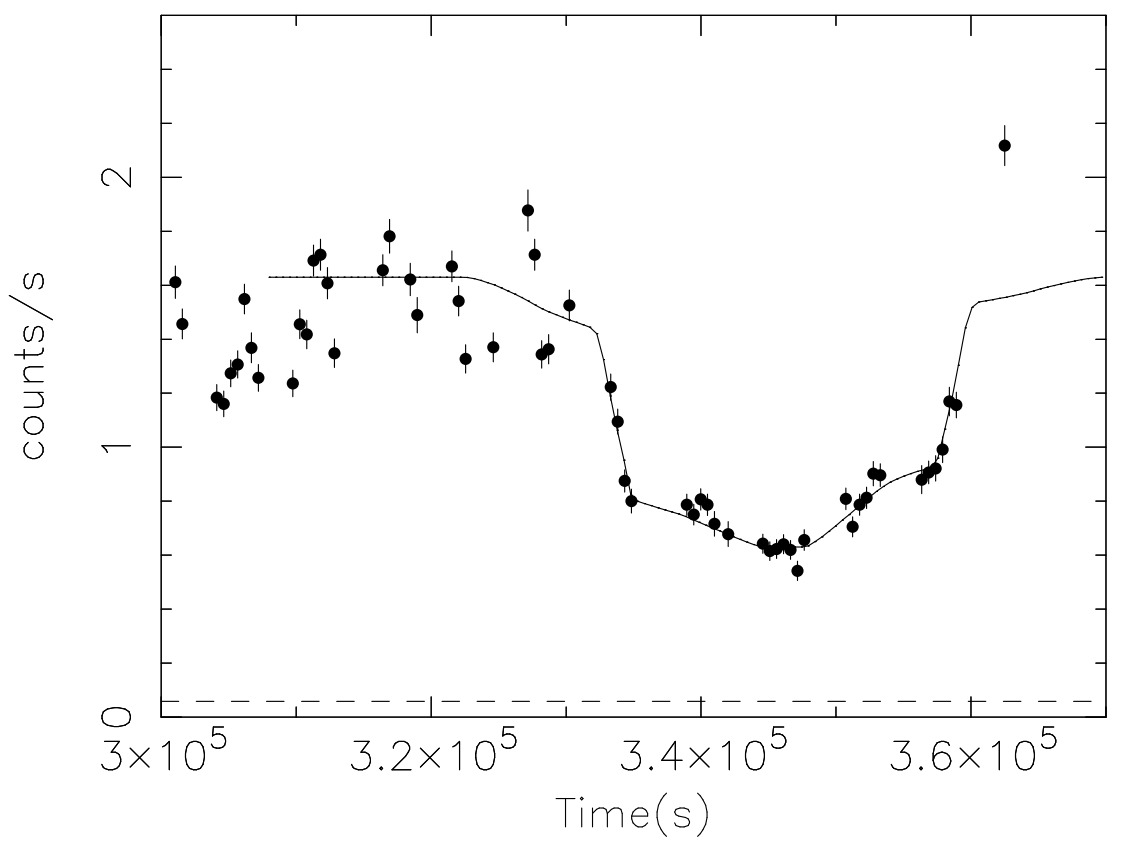

Fig. 2.- The deep minimum (DM) light curve with the model from McKernan and Yaqoob (1998) in which the temporal profile of the DM lightcurve can be explained by an obscuring body passing over the disk and central source. To explain the temporal data, the part of the disk for which the emission is blueshifted must be occulted first, and it remains occulted for a large part of the duration of the intensity dip. The dashed line is the mean background level. 


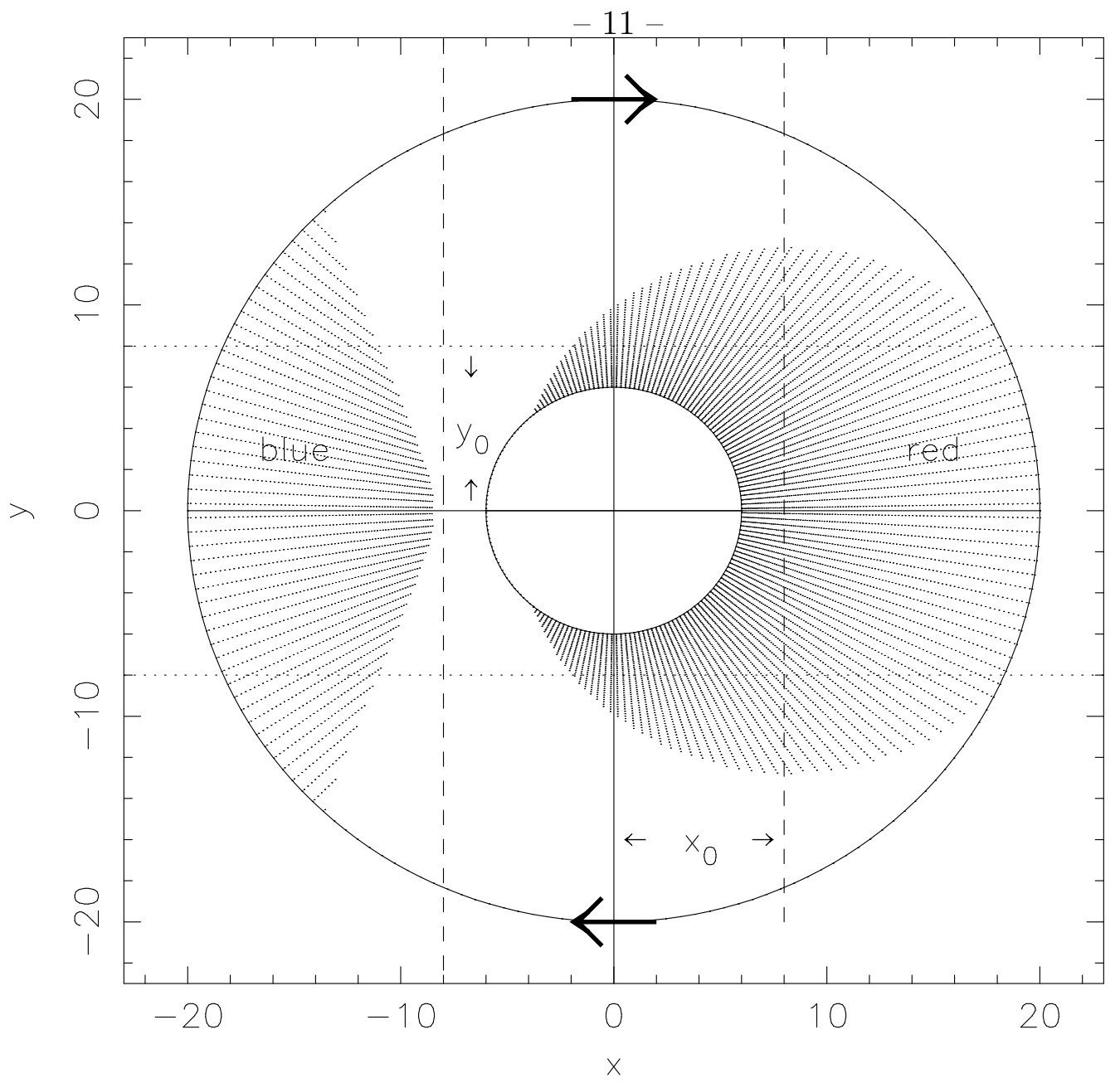

Fig. 3.- Illustration of how different parts of the Schwarzschild disk affect the Doppler and gravitational shift of line photons. Defining $g=E$ (observed) $/ E$ (emitted) (including both Doppler and gravitational effects), the shaded part labeled blue corresponds to $g>1$ and the shaded part labeled red corresponds to $g<(1 / 1.2)$. The obscuration model is such that lines-of-sight to the region inside $|x|<x_{0}$ or $|y|<y_{0}$ are blocked from view (see text). In this diagram the disk is inclined at $30^{\circ}$, such that the disk normal is pointing downwards towards the negative $y$ axis. The inner and outer disk radii are $r_{i}=6 r_{g}$ and $r_{o}=20 r_{g}$. 


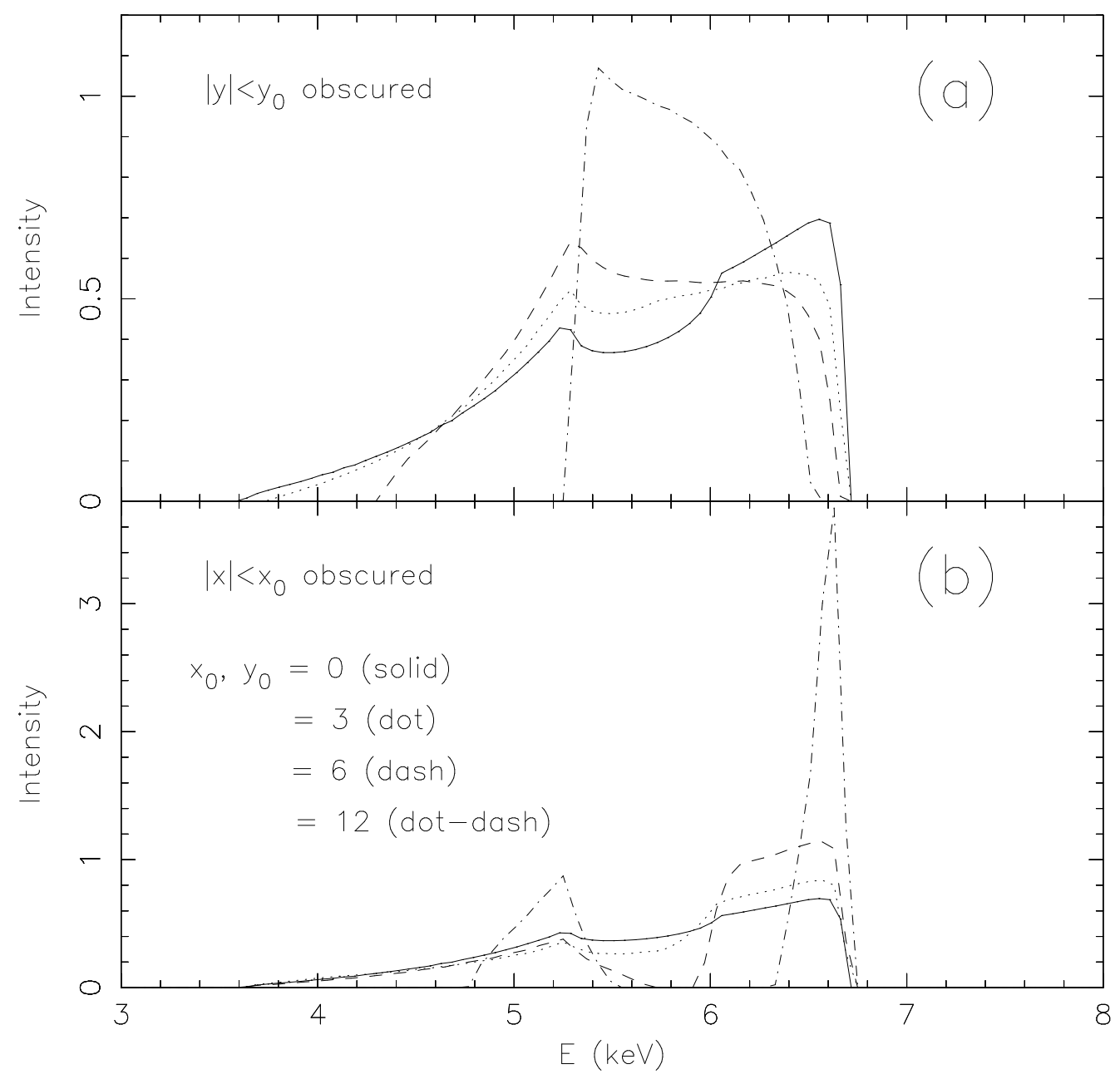

Fig. 4.- Theoretical calculations of the iron line profile from an obscured Schwarzschild Fig. 4 disk with the dimensions of the obscurer $\left(x_{0}\right.$ and $\left.y_{0}\right)$ as shown and the remaining parameters as in Figure 3. 


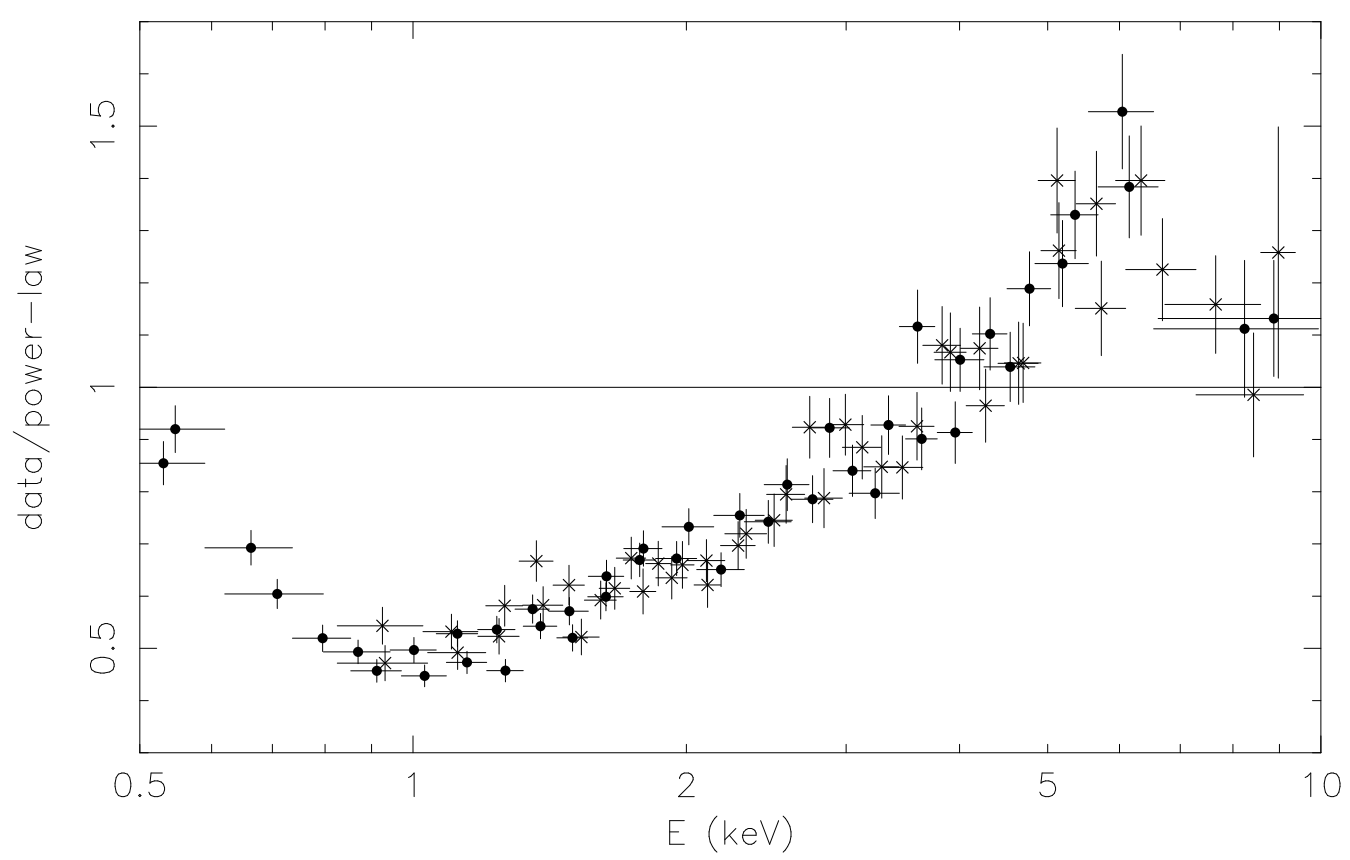

Fig. 5. - Ratio of the data to model when the deep minimum spectra (from the four $A S C A$ instruments) are fitted with only Galactic absorption and a power law with the slope fixed at the value obtained from the average 4.2-day spectrum (i.e., $\Gamma=1.98$, see model SH1 in Table 1). The extra absorption during the deep minimum is clearly evident, even above 3 $\mathrm{keV}$. Filled circles correspond to SIS0 and SIS1 data and crosses correspond to GIS2 and GIS3 data. 